\title{
Efficient Control Variates for Uncertainty Quantification of Radiation Transport
}

\author{
A. Frankel*, G. Iaccarino \\ Stanford University, 488 Escondido Mall, Building 500, Stanford, CA 94305
}

\begin{abstract}
Numerical simulations of problems involving radiation transport are challenging because of the associated computational cost; moreover, it is typically difficult to describe the optical properties of the system very precisely, and therefore uncertainties abound. We aim to represent the uncertainties explicitly and to characterize their impact on the output of interest. While stochastic collocation and polynomial chaos methods have been applied previously, these methods can suffer from the curse of dimensionality and fail in cases where the system response is discontinuous or highly nonlinear. Monte Carlo methods are more robust, but they converge slowly. To that end, we apply the control variate method to uncertainty propagation via Monte Carlo. We leverage the modeling hierarchy of radiation transport to use low fidelity models such as the diffusion approximation and coarse angular discretizations to reduce the confidence interval on the quantity of interest. The efficiency of the control variate method is demonstrated in several problems involving stochastic media, thermal emission, and radiation properties with different quantities of interest. The control variates are able to provide significant variance reduction and efficiency increase in all problems considered. We conclude our study with a discussion of choosing optimal control variates and other extensions of Monte Carlo methods.
\end{abstract}

\section{Introduction}

In many practical radiative heat transfer applications, we have limited information of the material properties of the system or specific knowledge of the thermal conditions of the environment. On the other hand, computational modeling requires direct specification of boundary conditions and identification of the physical parameters entering the governing equations. Uncertainty quantification (UQ) aims at bridging this gap by introducing a probabilistic description of the problem in which the thermal conditions, boundary conditions, and material properties are not specified precisely. Instead, we introduce stochastic variables informed by real observations that represent the related imprecision and methods for propagation of uncertainty through a computational or physical model. Common techniques for uncertainty propagation are classified into intrusive methods such as polynomial chaos, non-intrusive methods such as stochastic collocation, and Monte Carlo methods [1].

\footnotetext{
* Corresponding author

Email address: frankel1@stanford.edu (A. Frankel)
} 
Polynomial chaos is a deterministic approach involving the expansion of the uncertain variables into an orthogonal polynomial series expansion and the development of a strategy to evaluate the series coefficients which lead to a new set of governing equations $[2,3]$. It is a compact way of propagating the uncertainties, but it requires the development of a new computational tool to solve the resulting equations. Stochastic collocation is a non-intrusive alternative to polynomial chaos in which the uncertain variables are sampled at discrete collocation points, and the original computational model is solved at each point [4]. This technique does not require any additional models to be developed, but it does require many solutions of the original model. Both techniques can fail when the quantity of interest is a discontinuous or highly non-linear function of the input uncertainties.

Monte Carlo is the simplest and arguably the most robust way of handling high dimensional problems with complex responses, but it also converges very slowly. For $N$ independent and identically distributed samples of the inputs, the confidence interval on the expectation value of the quantity of interest decreases as $N^{-1 / 2}$. For problems requiring high accuracy or where the computational model is expensive to evaluate, this slow rate of convergence can be prohibitive. Consequently, researchers have employed variance reduction techniques to Monte Carlo to accelerate the convergence. One such method is the control variate method, in which a correlated model is used to correct the sample value to be closer to the mean $[5,6,7]$. Given a physical model, it is possible to identify simplifications that can be made to it that make it easier to solve without significantly reducing the correlation with the true model, and use the simplified model as a control variate

One possible application of the control variate approach is in the field of radiation transport or the closely related field of neutron transport. Uncertainty propagation in radiation transport has not been widely considered until very recently. [8, 9] used stochastic collocation to examine radiation transport in stochastic media, and [10] used polynomial chaos for similar problems in radiation and neutron transport. [11] used adjoint sensitivity analysis to propagate uncertainty in radiation spectral properties in hypersonic combustion. [12] considered the special case of a discrete mixture described by a renewal process and reduced the radiative transfer equation to a system of coupled equations for the ensemble averaged intensity. Each of these cases considered targeted the solution of the radiative transfer equation without using control variates. Radiation transport, however, has a rich modeling hierarchy in which lower fidelity models can be exploited to enhance the efficiency of solving higher fidelity models $[13,14]$.

In this work we will consider the application of the control variate method to radiation transport in a UQ setting. We will begin with an overview of the radiative transfer equation, the discrete ordinates method for solving it, and the diffusion approximation as a low order approximation. We will then demonstrate the control variate method and discuss the use of radiation models as control variates. Finally, we will demonstrate the success in variance reduction that may be achieved using these control variates in several problems involving stochastic media and radiative material properties. Although we limit ourselves to special classes of problems in radiation transport, the techniques discussed are easily extended to more complex systems and other related transport physics. 


\section{Monte Carlo Uncertainty Propagation}

To estimate the effects of uncertainties on a radiative quantity of interest, we use Monte Carlo methods, in which the input variables are randomly sampled and propagated into the physical model. If we denote the stochastic parameters of a system as $\vec{\zeta}$ and the quantity of interest as $Q$, we may estimate the expected value as

$$
E(Q)=\frac{1}{N} \sum_{i=1}^{N} Q_{i}
$$

for $N$ independently and identically distributed samples $\vec{\zeta}_{i}$ and function values $Q_{i}$ evaluated at each sample. The population variance may be estimated as

$$
V(Q)=\frac{1}{N-1} \sum_{i=1}^{N}\left(Q_{i}-E(Q)\right)^{2}
$$

and the standard error in the mean $s$ as

$$
s^{2}=\frac{V(Q)}{N}
$$

With these estimators, the confidence interval for the quantity of interest can be constructed using the central limit theorem. The $99 \%$ confidence interval is given as $\delta_{99}=3 s$, such that the true mean is in the interval $[E(Q)-3 s, E(Q)+3 s]$ with probability of $99 \%$.

The advantage of Monte Carlo sampling is that it can handle very high dimensional inputs without further modifications and without incurring any fundamental computational penalty; moreover, it does not require a smooth relation between the uncertain input and the quantity of interest. However, the confidence interval decreases quite slowly as $1 / \sqrt{N}$. Proper resolution of the quantity of interest may require many samples to converge to an acceptable range. This issue is particularly problematic if $Q$ is expensive to evaluate, i.e. if a lengthy computation must be performed in order to derive it.

\subsection{Variance Reduction}

One common approach to reduce the variance of a Monte Carlo computation is with the use of control variates $(\mathrm{CVs})$. While the classic approach involves the use of CVs with a known mean, we consider the case where the mean may not be known a priori here. Suppose there is a quantity $P$ which is linearly related to the quantity of interest $Q$. Then we may write the relationship directly as

$$
Q=\beta_{0}+\beta_{1} P
$$

for some coefficients $\beta_{0}$ and $\beta_{1}$. Then the expectation of $Q$ may be computed from

$$
E(Q)=\beta_{0}+\beta_{1} E(P)
$$

If $\beta_{1}$, and $E(P)$ are known, then given any one sample each $Q_{i}$ and $P_{i}$, the expectation of $Q$ can be recovered as

$$
E(Q)=Q_{i}-\beta_{1}\left(P_{i}-E(P)\right)
$$


If the relationship were exactly linear and $\beta_{1}$ and $E(P)$ were known, then one sample would suffice to recover $E(Q)$. In practice, it is difficult or impossible to obtain models of this form. However, this approach is the basis of the control variate Monte Carlo variance reduction technique. In the following discussion we follow the development of [6] with some simplifications specific to our particular case.

The sample values of the quantity of interest are shifted proportionately based on the value of the control variate. Thus we write a similar relation as above:

$$
\hat{Q}_{i}=Q_{i}+\alpha\left(P_{i}-E(P)\right)
$$

where $\hat{Q}_{i}$ is the modified sample value and $\alpha$ is a free coefficient. The intuition gained from the analysis above suggests that choosing a control variate $P$ that is highly correlated with the quantity of interest would yield values of $\hat{Q}$ that are closely centered around the true mean, thereby reducing the variance. We would also prefer to choose a model for $P$ that is simpler and easier to evaluate than $Q$ so that drawing many samples of it would not greatly increase the computational cost of the sampling procedure. Thus if $Q$ is a quantity derived from a model with high fidelity to the physics of interest, than $P$ could be a simpler representation of the same physics that is of lower fidelity but easier to evaluate. Thus we choose an approach where $Q$ is evaluated from a "high fidelity" model, and the control variate $P$ is evaluated from a "low fidelity" model.

In the case where $E(P)$ is not known a priori, it must be estimated using a separate sampling procedure. Suppose we allocate $N_{H}$ samples to compute samples $\hat{Q}$, and an additional $N_{L}$ samples to evaluate samples of $P$. Then we can write the estimators as

$$
\begin{gathered}
E(P)=\frac{1}{N_{H}+N_{L}} \sum_{i=1}^{N_{H}} P_{i}+\frac{1}{N_{H}+N_{L}} \sum_{j=1}^{N_{L}} P_{j} \\
E(Q)=\frac{1}{N_{H}} \sum_{i=1}^{N_{H}} Q_{i} \\
E(\hat{Q})=\frac{1}{N_{H}} \sum_{i=1}^{N_{H}} Q_{i}+\frac{\alpha N_{L}}{N_{H}\left(N_{H}+N_{L}\right)} \sum_{i=1}^{N_{H}} P_{i}-\frac{\alpha}{N_{H}+N_{L}} \sum_{j=1}^{N_{L}} P_{j}
\end{gathered}
$$

The question remains as to how to choose $\alpha$. Since $\alpha$ is a free variable, we may choose it to minimize the variance of $\hat{Q}$. For convenience, we also introduce the value $r=N_{L} / N_{H}$. The standard error estimator in this case is given by

$$
s^{2}=\frac{1}{N_{H}} V(Q)+\frac{\alpha^{2} r}{N_{H}(1+r)} V(P)+\frac{2 \alpha r}{N_{H}(1+r)} \operatorname{Cov}(P, Q)
$$

where the covariance $\operatorname{Cov}(P, Q)$ may be estimated from

$$
\operatorname{Cov}(P, Q)=\frac{1}{N_{H}-1} \sum_{i=1}^{N_{H}}\left(Q_{i}-E(Q)\right)\left(P_{i}-E(P)\right)
$$


With these definitions in place, the variance may be minimized by taking the derivative of 11 with respect to $\alpha$ and setting it to zero. The optimal value of $\alpha$ is

$$
\alpha=-\frac{\operatorname{Cov}(P, Q)}{V(P)}=-\rho \sqrt{\frac{V(Q)}{V(P)}}
$$

where $\rho$ is the correlation coefficient between the quantity of interest and the control variate:

$$
\rho=\frac{\operatorname{Cov}(P, Q)}{\sqrt{V(P) V(Q)}}
$$

Note that this definition of $\alpha$ is closely related with the least squares definition of a linear regression. With this definition in place, the standard error of the control variate samples is given by

$$
s^{2}=\left(1-\frac{r}{1+r} \rho^{2}\right) \frac{V(Q)}{N_{H}}
$$

Thus variance reduction is achieved with the satisfaction of two requirements: the choice of a control variate that is highly correlated with the quantity of interest (i.e. $\rho^{2} \approx 1$ ) and the ability to sample the control variate many times (i.e. $N_{L} \gg N_{H}$ ). As we will discuss in the following section, the computational cost of a single sample is also an important factor to consider in the selection of a control variate.

\subsection{Application to Radiation Transport}

In the context of radiation transport, we are primarily concerned with solutions for the radiation intensity $I$ with direction vector $\hat{s}$ of the gray and quasi-steady radiative transfer equation (RTE) with isotropic scattering [15]

$$
\hat{s} \cdot \nabla I=-\sigma_{e} I+(1-\omega) \sigma_{e} I_{b}+\frac{\omega \sigma_{e}}{4 \pi} \int_{4 \pi} I\left(\hat{s}^{\prime}\right) d \Omega
$$

subject to the diffuse wall boundary condition with wall normal $\hat{n}_{w}$

$$
I\left(\hat{s} \cdot \hat{n}_{w}>0, \vec{r}_{w}\right)=\epsilon_{w} I_{b w}+\frac{1-\epsilon_{w}}{\pi} \int_{\hat{s} \cdot \hat{n}_{w}<0} I\left|\hat{s} \cdot \hat{n}_{w}\right| d \Omega
$$

The extinction coefficient $\sigma_{e}$ and albedo $\omega$ may be uncertain, as well as the blackbody intensity $I_{b}$ and boundary emissivity $\epsilon$. Propagating these uncertainties in a Monte Carlo sampling approach is very computationally intensive, let alone computing a single solution of the RTE. As a result, it is common to rely on lower fidelity models like diffusion or coarse angular discretizations to approximate the behavior of radiation transport either as surrogate models or as solution preconditioners for high order discrete ordinates method (DOM) calculations [13]. In a similar fashion, we propose the use of the diffusion approximation [15] and low order DOM as control variates for high order DOM in Monte Carlo uncertainty propagation. These models are expected to be highly correlated with a high order DOM and show similar sensitivities to input uncertainties. Even though the use of a low fidelity model is expected to introduce an error into the solution, this is unimportant as the objective is to evaluate the variability of the estimates and, consequently, the correlation with 
the high fidelity model.

The remaining question is to decide how to choose a particular control variate for a given problem. Some choices may be particularly highly correlated with the high fidelity samples, but may be too expensive to be practical. Similarly, relatively cheap calculations may be poorly correlated. To quantify the effectiveness of a given control variate, we introduce the figure-of-merit fom:

$$
\text { fom }=\frac{1}{\delta^{2} t}
$$

where $\delta$ is the confidence interval given as before, and $t$ is the wall-clock time for a given computation. Since the confidence interval decreases as $1 / N$ but the time increases as $N$, the figure-of-merit is approximately invariant with respect to the number of samples taken. Thus it is not sufficient to report a reduced confidence interval for a given problem; a meaningful variance reduction occurs only if the confidence interval decreases more than the runtime increases. For the control variate approach, the runtime incorporates both the time taking the high fidelity samples as well as the control variate samples, so the runtime may increase dramatically if many control variate samples are required. The result is a tradeoff between choices of control variates: simpler control variates have lower correlation coefficients and would provide lower variance reduction, but are substantially cheaper to compute and may not increase the runtime more than they decrease the variance. Better control variates will have higher correlation coefficients, but they will be more expensive to evaluate.

The expectation is that for different problems there may be different optimal choices of control variates. For particularly simple problems (or for problems in optically thick media), the diffusion approximation may be an excellent candidate for a control variate. In problems that depend on more detailed information about the radiation intensity, a particularly high order DOM calculation may be optimal. In general, one may expect a low order DOM calculation to provide the balance between computational cost and correlation with the high fidelity calculation. Of course, in some multidimensional cases the ray effect may make low order DOM control variates unviable.

It is also possible to use multiple control variates simultaneously for a given problem. Such an approach could balance out the inaccuracy of a low fidelity control variate with the cost of a high fidelity control variate. For example, diffusion could be used to quickly capture most of the variation in the solution behavior with the uncertainties, and then a low order DOM calculation could be used to capture the additional transport effects not contained within the diffusion approximation. Of course many combinations are possible, each coming with an increased cost compared to computing a single control variate, and so we defer this consideration to a future study.

\section{Examples}

In the following sections we will consider specific cases of increasing relative computational difficulty where several stochastic inputs to a radiative system are present, and we will examine the efficacy of different choices of control variates. The DOM calculations are performed using a diamond differencing spatial discretization and a source iteration solver. $1 D$ simulations make use of a Gauss-Legendre quadrature computed from the Golub-Welsch algorithm [16, 17], and the $2 D$ simulations use the level symmetric quadrature sets $[15,18]$. The diffusion approximation is 


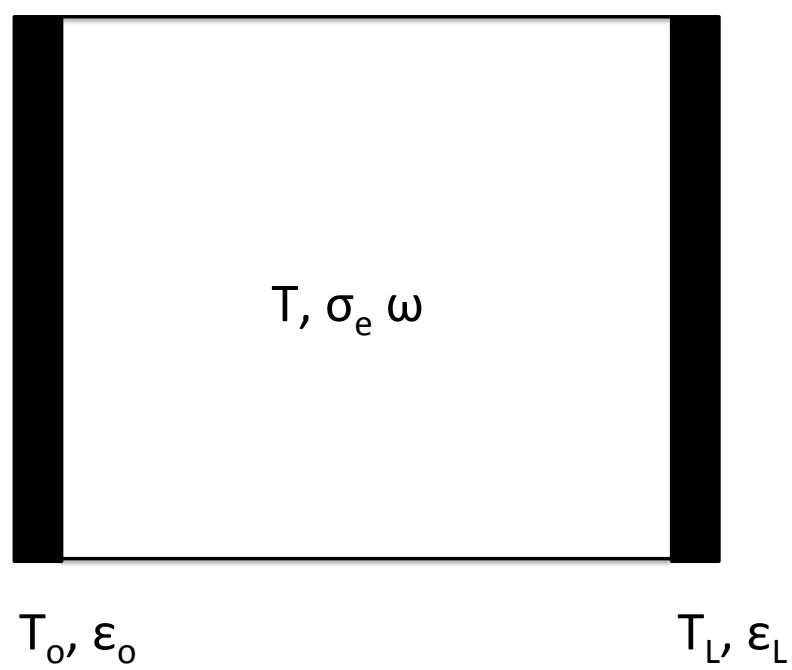

Figure 1: Geometry for examples 1 and 2

computed using second-order finite differences with the Marshak boundary condition.

\subsection{Example 1}

The first case under consideration involves radiation transport in a 1D slab with an uncertain temperature profile and extinction coefficient as shown in figure 1 . Note that $1 D$ refers to a spatially one-dimensional problem (with radiation variation in the $x$-direction), and with a laterally homogeneous system the dependence of the radiation on the $\eta$ angular coordinate disappears. Formally, the problem is two-dimensional, with $I=I(x, \xi)$.

The temperature profile and extinction coefficient are given by

$$
\begin{aligned}
& T(x)=T_{o} \zeta_{1}\left(1-\frac{x}{L}\right)^{2} \\
& \sigma_{e}(x)=\left\{\begin{array}{l}
0.1+50 \exp \left(-10 \zeta_{2}\right) m^{-1} \quad \frac{L}{4} \leq x \leq \frac{3 L}{4} \\
0.1 m^{-1} \quad \text { otherwise }
\end{array}\right.
\end{aligned}
$$

where $T_{o}=3000 \mathrm{~K}$ is the temperature of the left wall at $x=0, L=1 \mathrm{~m}$ is the length of the domain, and $\zeta_{1}$ and $\zeta_{2}$ are uniformly distributed random variables on the interval $[0,1]$. The slab is assumed to be non-scattering $(\omega=0)$, and surrounded by black walls $\left(\epsilon_{1}=\epsilon_{2}=1\right)$. Though the intensity has an analytical solution in this case [15], we use this problem to demonstrate the ability of the Monte Carlo uncertainty propagation technique to capture the solution. This problem could be considered an extremely simplified version of the transmission of solar radiation through the atmosphere, where the temperature of the atmosphere varies in space, and the clouds have a highly variable density. The quantities of interest represent a reduced-order description of the incident radiation, where the intensity field everywhere in space may be described by the mean $\left(Q_{1}\right)$ and a 
characteristic deviation $\left(Q_{2}\right)$.

In this setting, we consider the quantities of interest to be the spatially-averaged incident radiation,

$$
Q_{1}=\frac{1}{L} \int_{0}^{L} G(x) d x
$$

and the spatial standard deviation of the incident radiation

$$
Q_{2}=\sqrt{\frac{1}{L} \int_{0}^{L} G(x)^{2} d x-\left(\frac{1}{L} \int_{0}^{L} G(x) d x\right)^{2}}
$$

The number of samples for each case was taken to be constant. We began with a baseline brute force approach of Monte Carlo sampling with 1100 samples, and used this case to set the baseline figure-of-merit. We then used the control variate approach with $N_{H}=100$ of the brute force samples and $N_{L}=300$ for each control variate evaluated at the same samples. The reported fom for each case is taken relative to the baseline case value.

The high fidelity model was an 80-point Gauss-Legendre quadrature discrete ordinates calculation. The control variates used were the diffusion approximation with second-order spatial discretization and several low order discrete ordinates calculations with 2, 4, 8, and 16 point Gauss-Legendre quadratures. The spatial domain was discretized into 400 volumes in each case.

We also consider a simple control variate based on a linearization of the quantity of interest:

$$
P\left(\zeta_{1}, \zeta_{2}\right)=Q\left(\bar{\zeta}_{1}, \bar{\zeta}_{2}\right)+\nabla_{\zeta} Q \cdot \overrightarrow{\Delta \zeta}=Q\left(\bar{\zeta}_{1}, \bar{\zeta}_{2}\right)+\left.\frac{\partial Q}{\partial \zeta_{1}}\right|_{\bar{\zeta}_{1}, \bar{\zeta}_{2}}\left(\zeta_{1}-\bar{\zeta}_{1}\right)+\left.\frac{\partial Q}{\partial \zeta_{2}}\right|_{\bar{\zeta}_{1}, \bar{\zeta}_{2}}\left(\zeta_{2}-\bar{\zeta}_{2}\right)
$$

The partial derivatives are evaluated numerically using a second-order central finite difference about the average value of the uncertain inputs ( 0.5 for the uniform distribution case). Though not physically accurate, this control variate only requires 5 high-order function evaluations ( 1 for the average value, and 2 each for the partial derivatives), and subsequent evaluations are inexpensive. Thus in spite of the reduced global accuracy, the linear control variate may be a valuable alternative strategy.

Table 1 shows the results from the Monte Carlo uncertainty propagation problem in one particular instance. All of the control variates considered were able to reduce the confidence interval by about a factor of two (and hence the variance by a factor of four), and they were able to do so in less time than it would have taken to sample four times as many of the high fidelity model. This is because each of the control variates had over $99 \%$ correlation with the high fidelity model, with the exception of the linearized model. Based on the figure-of-merit, it appears that the diffusion approximation is the optimal choice for variance reduction, even though the correlation coefficient is slightly lower than the higher order discrete ordinates control variates. This is due to the relative cost of inverting the tridiagonal matrix from the 1D diffusion approximation compared to the discrete ordinates sweeping process. It should also be noted that the diffusion and $S_{2}$ models should theoretically be identical, save for the choice of boundary conditions. Different boundary conditions and discretizations can produce slightly different results, and the solution cost for diffusion is lower than that of the $S_{2}$ approximation, thus producing the differences shown in the table. It is noteworthy that the linearized model performed very well in spite of the lower correlation coefficient; 


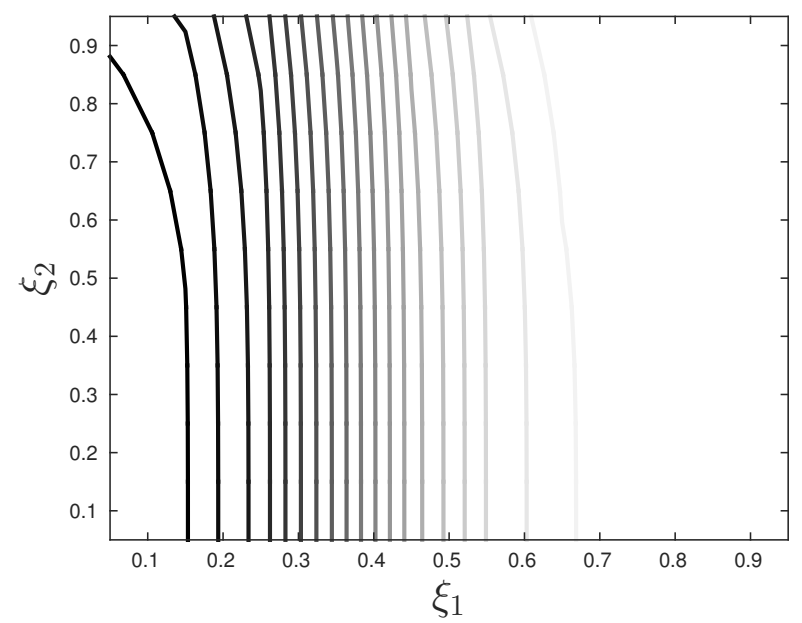

Figure 2: The contours for example 1 with quantity of interest $Q_{1}$. Lighter shades indicated larger values of $Q_{1}$.

this is due to the lower runtime per function evaluation compared to the other models. This also explains the relative performance of $S_{4}$ and $S_{2}$; despite the fact that the $S_{4}$ control variate is twice as expensive as $S_{2}$, the added runtime to the total cost is negligible compared to the cost of the $S_{80}$ simulations, and so the fom did not change substantially.

The success of these control variates is not necessarily due to using a simple quantity of interest. Figure 2 shows the contours of the quantity of interest as a function of the two random variables. The quantity of interest shows a non-linear response to the input variables, especially with respect to the temperature variable. A linear control variate would perform poorly in this case. However, the input of the random variables into the control variates produces a nearly linear relation with the high fidelity model, as demonstrated by the high value of the correlation coefficients.

\begin{tabular}{|c|c|c|c|c|c|c|c|c|}
\hline & baseline & no CV & diffusion & $S_{2}$ & $S_{4}$ & $S_{8}$ & $S_{16}$ & linearization \\
\hline$E(Q) \times 10^{6}\left(\mathrm{~W} / \mathrm{m}^{2}\right)$ & 5.5790 & 5.5463 & 5.5695 & 5.5693 & 5.5659 & 5.5676 & 5.5697 & 5.6328 \\
\hline$\delta \times 10^{5}\left(\mathrm{~W} / \mathrm{m}^{2}\right)$ & 1.8968 & 6.4537 & 3.2352 & 3.2422 & 3.2284 & 3.2278 & 3.2273 & 3.5808 \\
\hline$\delta / E(Q) \times 100 \%$ & 3.40 & 11.64 & 5.81 & 5.82 & 5.80 & 5.80 & 5.79 & 6.36 \\
\hline$\rho$ & - & - & 0.9991 & 0.9984 & 0.9998 & 0.9999 & 1.0000 & 0.9607 \\
\hline fom & 1 & 0.9505 & 3.7359 & 3.6748 & 3.6361 & 3.4556 & 3.0255 & 2.7244 \\
\hline$N_{H}$ & 1100 & 100 & 100 & 100 & 100 & 100 & 100 & 100 \\
\hline$N_{L}$ & 0 & 0 & 300 & 300 & 300 & 300 & 300 & 300 \\
\hline
\end{tabular}

Table 1: Results from a particular run of the MC uncertainty propagation with various control variates for example 1 for the quantity of interest $Q_{1}$. The "baseline" case is a brute-force Monte Carlo run taken to establish a higher accuracy estimate of the mean and provide a figure-of-merit for comparison. The "no CV" case is 100 samples taken without using a control variate. All control variate cases are taken with the same samples as the "no CV" case.

Table 2 shows the results for the second quantity of interest. The correlation coefficient and figure-of-merit show the same trend as in the previous case, though here the relative error is significantly larger, especially for the case with 100 samples and no control variate. As in the previous 


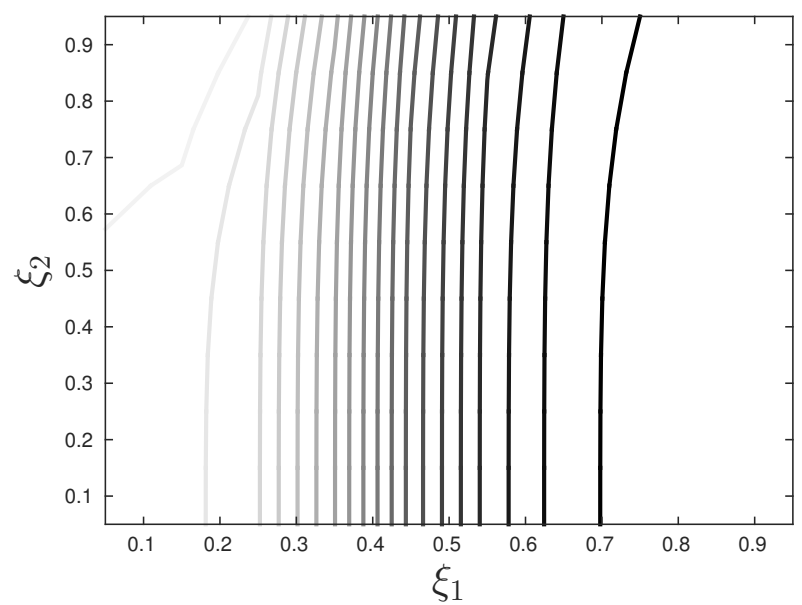

Figure 3: The contours for example 1 with quantity of interest $Q_{2}$. Lighter shades indicated larger values of $Q_{2}$.

case, the diffusion control variate appears to be the best choice for variance reduction, although all of the radiation-based control variates were able to provide a factor of two reduction in the confidence interval. We note from the contour plots that the response is non-linear with respect to the random variables as in the previous problem, and only through the transformation in the control variates is a strongly linear response recovered.

\begin{tabular}{|c|c|c|c|c|c|c|c|c|}
\hline & baseline & no CV & diffusion & $S_{2}$ & $S_{4}$ & $S_{8}$ & $S_{16}$ & linearization \\
\hline$E(Q) \times 10^{6}\left(\mathrm{~W} / \mathrm{m}^{2}\right)$ & 2.1011 & 2.1925 & 2.1311 & 2.1322 & 2.1233 & 2.1241 & 2.1268 & 2.1454 \\
\hline$\delta \times 10^{5}\left(\mathrm{~W} / \mathrm{m}^{2}\right)$ & 1.1382 & 3.6392 & 1.8310 & 1.8283 & 1.8229 & 1.8219 & 1.8203 & 2.0576 \\
\hline$\delta / E(Q) \times 100 \%$ & 5.42 & 16.60 & 8.59 & 8.58 & 8.59 & 8.58 & 8.56 & 9.59 \\
\hline$\rho$ & - & - & 0.9979 & 0.9984 & 0.9994 & 0.9996 & 0.9999 & 0.9524 \\
\hline fom & 1 & 1.0750 & 4.1857 & 4.1478 & 4.0921 & 3.8921 & 3.4106 & 2.9667 \\
\hline
\end{tabular}

Table 2: Results from a particular run of the MC uncertainty propagation with various control variates for example 1 for the quantity of interest $Q_{2}$. The numbers of runs and explanations are found in table 1.

\subsection{Example 2}

In this section we consider the problem of radiation transport in one-dimensional stochastic media. Stochastic media appear in many different physical applications, including pebble-bed nuclear reactors, atmospheric physics with random cloud distributions, and sooting flames. In some special cases, the governing equations may be modified to account for the medium stochasticity, but in general this problem may require Monte Carlo simulations where different realizations of the media are sampled. As an example of such a problem, we prescribe an extinction coefficient governed approximately by a Gaussian process with an exponential covariance function. To generate these extinction coefficients, we construct a zero-mean $\operatorname{AR}(1)$ process $s(x)$ as

$$
s_{i+1}=r s_{i}+\sqrt{1-r^{2}} \sigma Z_{i+1}
$$



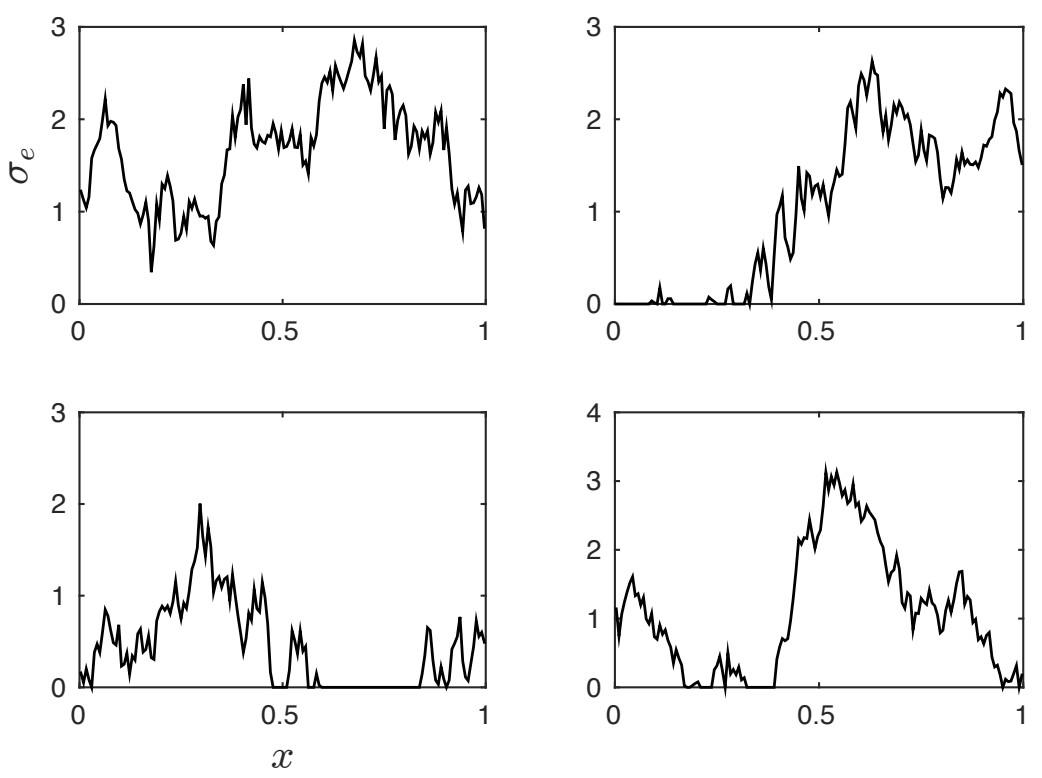

Figure 4: Realizations of the extinction coefficient field for example 2.

where $r=\operatorname{Cov}\left(\sigma_{e, i}, \sigma_{e, i+1}\right)$ is the covariance function, $\sigma$ is the standard deviation of the extinction coefficient, and $Z_{i} \sim \mathcal{N}(0,1)$ is a normally distributed random variable with zero mean and variance of 1 . The exponential covariance function is set such that $r=\exp (-\Delta x / l)$ with correlation length $l$ and step size $\Delta z$. It can be readily verified that $E(s)=0, V(s)=\sigma^{2}$, and $\rho\left(s_{i+1}, s_{i}\right)=\exp (-\Delta x / l)$ as desired for the Gaussian process construction. The field is initialized with a value of $s_{1}=\sigma Z_{1}$, and a chain of $N_{z}=150$ values is generated. After the field has been generated, the desired mean $\mu$ is added to the extinction coefficient:

$$
\sigma_{e}(x)=\mu+s(x)
$$

Finally, since the extinction coefficient from this process may be negative, values less than or equal to zero are set to be a small positive value (e.g. $10^{-8}$ ). This is to both prevent unphysical and numerically unstable results from being generated as well as to provide regularization for the diffusion approximation, in which the diffusion coefficient scales as $1 / \sigma_{e}$. The result is a spatially variable extinction field as a random field, i.e. there are $N_{x}$ uncertainties as inputs to the problem.

In this example, we take the same quantities of interest and configuration as in the previous example, but do not consider a linearized model due to the relative difficulty in constructing such a model. The scattering albedo is set to $\omega=0$, and as before the boundaries are set to be black. The extinction coefficient was set to have a nominal mean $1 \mathrm{~m}^{-1}$, standard deviation $1 \mathrm{~m}^{-1}$, and correlation length $0.3 \mathrm{~m}$. With these parameters, approximately $17 \%$ of the extinction coefficient values in all cases required regularization. Example realizations of this random process may be seen in figure 4. The temperature of the left wall was $T=3000 K$, the temperature of the right wall was $0 K$, and the temperature of the medium was also uniformly $0 K$. The same high fidelity model and control variates were used as in the previous problem, along with the same numbers of samples. 


\begin{tabular}{|c|c|c|c|c|c|c|c|}
\hline & baseline & no CV & diffusion & $S_{2}$ & $S_{4}$ & $S_{8}$ & $S_{16}$ \\
\hline$E(Q) \times 10^{6}\left(\mathrm{~W} / \mathrm{m}^{2}\right)$ & 4.0362 & 3.8244 & 3.9601 & 3.9621 & 3.9519 & 3.9409 & 3.9358 \\
\hline$\delta \times 10^{5}\left(\mathrm{~W} / \mathrm{m}^{2}\right)$ & 1.4617 & 4.9782 & 2.5268 & 2.5351 & 2.4973 & 2.4906 & 2.4895 \\
\hline$\delta / E(Q) \times 100 \%$ & 3.62 & 13.02 & 6.38 & 6.40 & 6.32 & 6.32 & 6.33 \\
\hline$\rho$ & - & - & 0.9949 & 0.9938 & 0.9989 & 0.9998 & 0.9999 \\
\hline fom & 1 & 0.9478 & 3.6339 & 3.5662 & 3.6156 & 3.4719 & 3.0571 \\
\hline
\end{tabular}

Table 3: Results from a particular run of the MC uncertainty propagation with various control variates for example 2 with quantity of interest $Q_{1}$. The numbers of runs and explanations are found in table 1.

Table 3 shows example results from a run for the first quantity of interest. As in the previous example, all of the control variates are highly correlated with the high fidelity model, and significant variance reduction is achieved for all of the control variates tested. The diffusion approximation is highly correlated and has a low computational cost, and thus has the highest figure-of-merit. This is due to the choice of quantity of interest; the integral measure of the radiation field causes a cancellation of errors in the higher frequency modes of the incident radiation behavior and is thus most strongly dependent on the behavior of the low frequency modes. Since the diffusion approximation captures the low frequency modes most accurately [13], it is expected to carry a high correlation with the high order discrete ordinates.

\begin{tabular}{|c|c|c|c|c|c|c|c|}
\hline & baseline & no CV & diffusion & $S_{2}$ & $S_{4}$ & $S_{8}$ & $S_{16}$ \\
\hline$E(Q) \times 10^{6}\left(\mathrm{~W} / \mathrm{m}^{2}\right)$ & 1.9176 & 1.9107 & 1.9664 & 1.9646 & 1.9538 & 1.9445 & 1.9432 \\
\hline$\delta \times 10^{5}\left(\mathrm{~W} / \mathrm{m}^{2}\right)$ & 0.4609 & 1.5440 & 0.9256 & 0.9092 & 0.8110 & 0.7807 & 0.7737 \\
\hline$\delta / E(Q) \times 100 \%$ & 2.40 & 8.08 & 4.71 & 4.63 & 4.15 & 4.02 & 3.98 \\
\hline$\rho$ & - & - & 0.9242 & 0.9333 & 0.9826 & 0.9962 & 0.9993 \\
\hline fom & 1 & 0.9778 & 2.6791 & 2.7432 & 3.3906 & 3.4960 & 3.1351 \\
\hline
\end{tabular}

Table 4: Results from a particular run of the MC uncertainty propagation with various control variates for example 2 with quantity of interest $Q_{2}$. The numbers of runs and explanations are found in table 1.

The results for the second quantity of interest are reported in table 4 . The correlation coefficients in this case are lower, indicating that the control variates do not capture the sensitivity of the radiation field for this particular quantity of interest. However, the variance reduction is comparable to the previous quantity of interest, with a lower relative error and a factor of 2 reduction in the confidence interval. The figure-of-merit also shows a clear tradeoff in variance reduction versus computational expense, where across the different models the figure-of-merit increases due to the increased correlation coefficient, and then decreases due to the increasing computational cost. The optimal control variate in this case appears to be the $S_{8}$ control variate, indicating that a low order discrete ordinates computation may be more appropriate as a control variate for a quantity of interest that is more sensitive to the spatial structure of the radiation intensity.

\subsection{Example 3}

In this final example, we apply the control variate technique to radiation transport in a twodimensional rectangular enclosure with scattering as shown in figure 5 . The discrete ordinates 


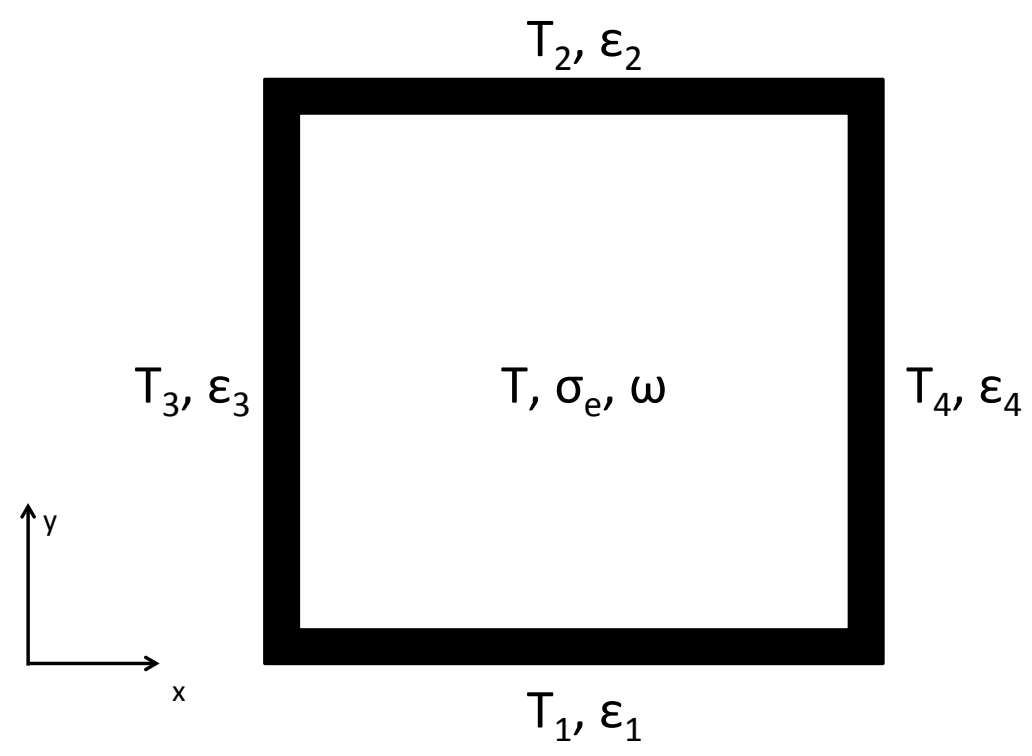

Figure 5: Geometry for example 3.

method may suffer from a loss of accuracy due to the presence of unphysical oscillations in the incident radiation and heat flux as a result of the ray effect [19]. The higher dimensionality in physical and angular space will also raise the cost of the control variates, and the addition of scattering will increase the cost of the discrete ordinates solutions, making the computational time a more critical factor.

The domain is a square with dimensions $1 m \times 1 m$ and 100 volumes in each direction. The extinction coefficient is given by

$$
\sigma_{e}(x, y)=10 \zeta_{1}(x(L-x)+y(L-y))
$$

The albedo is uniform and uncertain:

$$
\omega=\zeta_{2}
$$

Finally, the two side walls have uncertain emissivities:

$$
\epsilon_{3}=\epsilon_{4}=\zeta_{3}
$$

The temperature of wall 1 is set to $3000 K$, and the other walls and the medium are all set to $300 K$. Walls 1 and 2 are both black. We consider two quantities of interest here: the spatiallyaveraged normal heat flux to wall 2 , and the spatial standard deviation of the normal heat flux to wall 2:

$$
\begin{gathered}
Q_{1}=\frac{1}{L} \int_{0}^{L} \vec{q}(x, y=L) \cdot \hat{y} d x \\
Q_{2}=\sqrt{\frac{1}{L} \int_{0}^{L}(\vec{q}(x, y=L) \cdot \hat{y})^{2} d x-\left(\frac{1}{L} \int_{0}^{L} \vec{q}(x, y=L) \cdot \hat{y} d x\right)^{2}}
\end{gathered}
$$

This problem setting is representative of a furnace where the medium is concentrated in the center, and the radiation properties of the medium and the walls are poorly characterized, and we 
are interested in the heating of the side walls. To solve the problem, we use the level-symmetric quadrature sets to discretize the angular domain, choosing $S_{16}$ (144 angles) to be the high fidelity model. The control variates used were the diffusion approximation and lower order level-symmetric quadratures $S_{2}$ (4 angles), $S_{4}$ (12 angles), $S_{6}$ (24 angles), and $S_{8}$ (40 angles). As in the previous examples, we use $N_{H}=100$ and $N_{L}=300$, and run an additional 1000 high fidelity samples to resolve a baseline case. We also consider an additional linearized model, similar to example 1, in which the gradient of the quantity of interest is evaluated using a second-order central difference for each of the partial derivatives, and the base value is evaluated at the average value for each of the uncertain variables. In total, the linearized model requires 7 high fidelity function evaluations.

Table 5 shows the results for the first quantity of interest in a sample run. The $S_{2}$ approximation clearly suffers from a loss of accuracy in this setting due to the onset of ray effects in the solution, but still has a high correlation with the high fidelity model. Even though the higher order discrete ordinates control variates have higher correlation, the diffusion approximation has the highest figure-of-merit due to the low cost of solving the system. In all cases considered, the control variate approach yielded a significant variance reduction. The contours of $Q_{1}$ for this case shown in figure 6 also show that the problem is strongly non-linear in each of the three random variables, and a linear control variate would be unable to capture the behavior of the radiation.

\begin{tabular}{|c|c|c|c|c|c|c|c|c|}
\hline & baseline & no CV & diffusion & $S_{2}$ & $S_{4}$ & $S_{6}$ & $S_{8}$ & linearization \\
\hline$E(Q) \times 10^{5}\left(\mathrm{~W} / \mathrm{m}^{2}\right)$ & 7.1501 & 8.5475 & 6.9499 & 7.1381 & 6.8775 & 6.8845 & 6.8840 & 6.7598 \\
\hline$\delta \times 10^{5}\left(\mathrm{~W} / \mathrm{m}^{2}\right)$ & 0.5742 & 1.8198 & 0.9211 & 1.1684 & 0.9118 & 0.9099 & 0.9103 & 1.1180 \\
\hline$\delta / E(Q) \times 100 \%$ & 8.03 & 21.29 & 13.25 & 16.37 & 13.26 & 13.22 & 13.22 & 16.54 \\
\hline$\rho$ & - & - & 0.9959 & 0.8852 & 0.9993 & 1.0000 & 0.9999 & 0.9111 \\
\hline fom & 1 & 1.0402 & 3.9792 & 2.3195 & 3.1947 & 2.5858 & 2.0291 & 2.6281 \\
\hline
\end{tabular}

Table 5: Results from a particular run of the MC uncertainty propagation with various control variates for example 3 with quantity of interest $Q_{1}$. The numbers of runs and explanations are found in table 1.

Table 6 shows the results for the second quantity of interest. The magnitude of the correlation coefficient for the diffusion and $S_{2}$ approximations suggests that those control variates cannot capture the physics of the problem accurately enough to provide variance reduction since $\rho^{2} \ll 1$ in both cases. The diffusion approximation cannot capture the variability of the heat flux in space since the intensity is only linear-anisotropic, and the $S_{2}$ approximation has the same limitation in addition to the ray effect. It is clear from the trend in the figure-of-merit that the tradeoff in accuracy versus computational effort leads to an optimal selection of $S_{4}$ as the control variate. This result shows that the quantity of interest can have a dramatic impact on the choice of control variate, and some caution must be taken in model selection before beginning a UQ investigation. The contours shown in figure 7 are more complex and show a greater degree of non-monotonicity than for $Q_{1}$, further demonstrating the challenging problem presented here.

\section{Conclusion}

We have demonstrated that the control variate method can be applied in an uncertainty quantification study of radiation transport. If the high fidelity model chosen is a well-resolved solution 

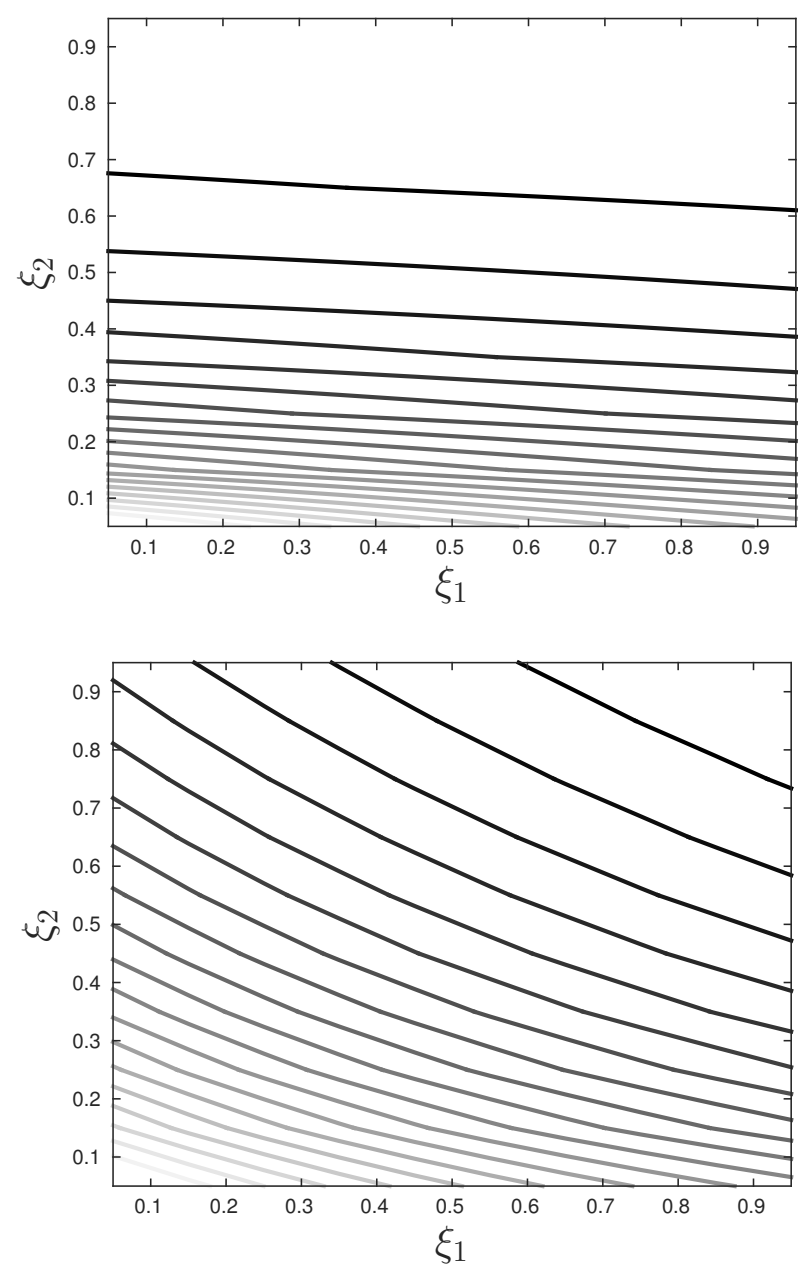

Figure 6: The contours for example 3 with quantity of interest $Q_{2}$ at $\xi_{3}=0.05$ (top) and $\xi_{3}=0.95$ (bottom). Lighter shades indicated larger values of $Q_{2}$. 

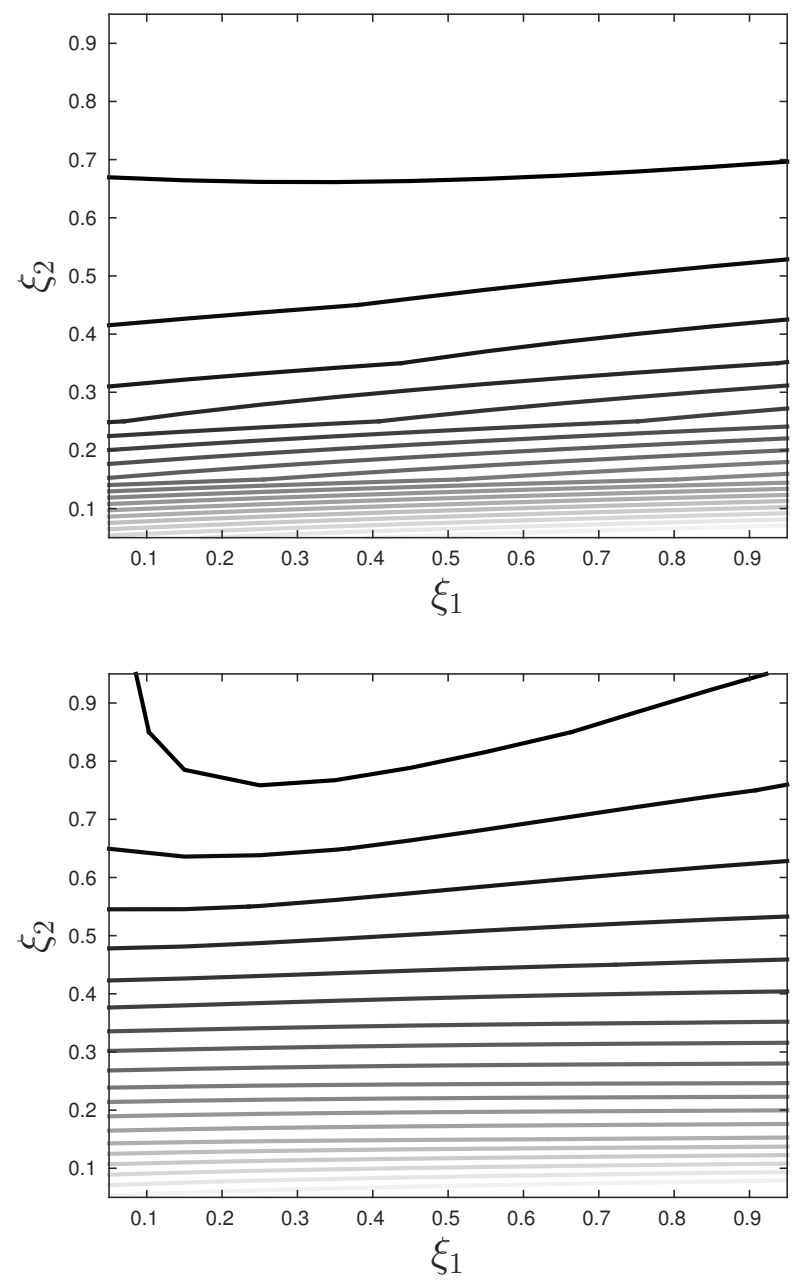

Figure 7: The contours for example 3 with quantity of interest $Q_{2}$ at $\xi_{3}=0.05$ (top) and $\xi_{3}=0.95$ (bottom). Lighter shades indicated larger values of $Q_{2}$. 


\begin{tabular}{|c|c|c|c|c|c|c|c|c|}
\hline & baseline & no CV & diffusion & $S_{2}$ & $S_{4}$ & $S_{6}$ & $S_{8}$ & linearization \\
\hline$E(Q) \times 10^{4}\left(\mathrm{~W} / \mathrm{m}^{2}\right)$ & 5.5560 & 5.8741 & 5.9774 & 5.7291 & 5.2871 & 5.3604 & 5.2400 & 5.1064 \\
\hline$\delta \times 10^{4}\left(\mathrm{~W} / \mathrm{m}^{2}\right)$ & 0.4794 & 1.5168 & 1.4888 & 1.4116 & 0.8262 & 0.7912 & 0.7694 & 1.0376 \\
\hline$\delta / E(Q) \times 100 \%$ & 8.63 & 25.82 & 24.91 & 24.64 & 15.63 & 14.76 & 14.68 & 20.32 \\
\hline$\rho$ & - & - & -0.2207 & -0.4226 & 0.9684 & 0.9852 & 0.9951 & 0.8423 \\
\hline fom & 1 & 1.0697 & 1.0875 & 1.1324 & 2.7576 & 2.4133 & 1.9942 & 2.1769 \\
\hline
\end{tabular}

Table 6: Results from a particular run of the MC uncertainty propagation with various control variates for example 3 with quantity of interest $Q_{2}$. The numbers of runs and explanations are found in table 1.

of the radiative transfer equation by the discrete ordinates method, then a lower fidelity model can be selected as a control variate. The lower fidelity models explored in this work were the diffusion approximation and lower order discrete ordinates calculations. The same idea can be extended to other choices of high and low fidelity. Any convergent solution of the radiative transfer equation may qualify as the high fidelity model. Thus discrete ordinates, Monte Carlo ray tracing, and the method of spherical harmonics, all carried out to sufficient spatial and angular resolution, may be used. Then it is possible to choose a high order spherical harmonics expansion as the high fidelity model and a low order expansion as the low fidelity, or Monte Carlo ray tracing as the high fidelity and discrete ordinates as the low fidelity. Although our results have shown that a linearized model may also be used successfully, we note that each additional uncertain variable requires additional high fidelity model evaluations to approximate the derivative, which may become prohibitive in cases where the high fidelity model is extremely expensive and there are many uncertain variables.

The diffusion approximation is a commonly used surrogate for radiation transport, and the lower order discrete ordinates can use the same code as the higher order discrete ordinates with little to no additional computational overhead, so these selections are appropriate candidates for consideration. Many other choices are possible, though. For example, flux-limited diffusion approximations are known to be accurate across a wider range of optical depths and are thus bound to be more accurate, but come at the cost of solving a non-linear Helmholtz equation. In the lower order discrete ordinates category, it is possible to modify the equations to use a smaller effective scattering albedo and/or use a fixed number of iterations in the solution to reduce the cost, but these modifications result in a loss of accuracy.

An open question resulting from our study is the problem of choosing a control variate. We have shown that depending on the problem under consideration and the quantity of interest, different control variates will have varying levels of success both in terms of computational cost and maintaining high correlation. It is not always possible to know which control variate will be optimal a priori. With expert knowledge one may guess that certain control variates may or may not be successful for a given problem (for example, we were able to guess that ray effects might interfere with the solution quality in example 3 above). However, this is not sufficient for selecting an optimal control variate since the precise balance of cost and accuracy cannot be known. One simple approach for solving this problem is to perform a pilot run of the problem under consideration with multiple control variates with a small number of samples to construct coarse estimates of the computational time and correlation coefficient, and based on that pilot run to select an optimal control variate for use in the problem. In the event that such a trial run is infeasible, we have 
shown that one should still choose a low order DOM control variate; even though it is unlikely to be optimal, it should still provide useful variance reduction.

Other potential opportunities for variance reduction exist in this setting. The multilevel Monte Carlo technique can also be applied to allow for coarser spatial discretizations, and in the context of the discrete ordinates method it can also be used to coarser angular discretizations. The multilevel and multifidelity techniques can be combined to use correlations both in lower fidelity and coarser discretization with the high fidelity, fine discretization model [6]. Many combinations are possible in radiation transport due to the modeling hierarchy and various levels of discretization available in the angular domain.

\section{Acknowledgements}

This work was funded by the Predictive Sciences Academic Alliance Program 2 at Stanford University.

\section{References}

[1] H. Najm. Uncertainty quantification and polynomial chaos techniques in computational fluid dynamics. Annu. Rev. Fluid Mech., 41:35-52, 2009.

[2] D. Xiu and G. Karniadakis. Modeling uncertainty in flow simulations via generalized polynomial chaos. J. Comp. Phys., 187:137-167, 2003.

[3] D. Xiu and G. Karniadakis. The Wiener-Askey polynomial chaos for stochastic differential equations. J. Sci. Comput., 24:619-644, 2006.

[4] I. Babuska, F. Nobile, and R. Tempone. A stochastic collocation method for elliptic partial differential equations with random input data. J. Numer. Anal., 45:1005-1034, 2007.

[5] Peter W. Glynn and Roberto Szechtman. Some New Perspectives on the Method of Control Variates, pages 27-49. Springer Berlin Heidelberg, Berlin, Heidelberg, 2002.

[6] G. Geraci, M. Eldred, and G. Iaccarino. A multifidelity control variate approach for the multilevel Monte Carlo technique. CTR Annual Research Briefs, pages 169-181, 2015.

[7] A. Padron, J. Alonso, F. Palacios, M. Barone, and M. Eldred. Multi-fidelity uncertainty quantification: Application to a vertical axis wind turbine under an extreme gust. Proc. of the 15th AIAA/ISSMO Multidisciplinary Analysis and Optimization Conference, 2014.

[8] E. Fichtl and A. Prinja. The stochastic collocation method for radiation transport in random media. J. Quant. Spec. and Rad. Trans., pages 646-659, 2011.

[9] A. Emery. Some thoughts on solving the radiative transfer equation in stochastic media using polynomial chaos and Wick products as applied to radiative equilibrium. J. Quant. Spec. and Rad. Trans., pages 61-77, 2005. 
[10] E. Fichtl, A. Prinja, and J. Warsa. Stochastic methods for uncertainty quantification in radiation transport. International Conference on Mathematics, Computational Methods 85 Reactor Physics, 2009.

[11] A. Crow. Computational Uncertainty Quantification of Thermal Radiation in Supersonic Combustion Chambers. PhD thesis, University of Michigan, 2013.

[12] G. Pomraning. Linear Kinetic Theory and Particle Transport in Stochastic Mixtures. World Scientific, 1991.

[13] M. Adams and E. Larsen. Fast iterative methods for discrete ordinates particle transport calculations. Progress in Nuclear Energy, pages 3-159, 2002.

[14] J. Densmore, H. Park, A. Wollaber, R. Rauenzahn, and D. Knoll. Monte carlo simulation methods in moment-based scale-bridging algorithms for thermal radiative-transfer problems. J. Comp. Phys., 284:40-58, 2015.

[15] M. Modest. Radiative Heat Transfer. Academic Press, 3rd edition, 2013.

[16] G. Golub and J. Welsch. Calculation of Gauss quadrature rules. Math. Comp., 23:221-230, 1969.

[17] L. Trefethen. Is Gauss quadrature better than Clenshaw-Curtis? SIAM, 50:67-87, 2008.

[18] E. Lewis and W. Miller. Computational methods of neutron transport. American Nuclear Society, 1993.

[19] E. Larsen and A. Wollaber. A quantitative theory of angular truncation errors in threedimensional $S_{N}$ calculations. Nuclear Science and Engineering, 160:267-283, 2008. 